\title{
Quantitatively determining the total bacterial count of raw goat milk using dielectric spectra
}

\author{
Zhuozhuo Zhu, Xinhua Zhu, Fanrong Kong, and Wenchuan Guo* \\ College of Mechanical and Electronic Engineering, Northwest A\&F University, Yangling, Shaanxi, 712100, China
}

\begin{abstract}
The objective of this study was to evaluate dielectric spectra as a means of quantitatively determining total bacterial count (TBC) of raw goat milk. The dielectric spectra, including dielectric constant $\left(\varepsilon^{\prime}\right)$ spectra and dielectric loss factor $\left(\varepsilon^{\prime \prime}\right)$ spectra, and TBC of 154 raw goat milk samples were measured using network analyzer and plate count methods, respectively. Owing to the poor linear relationship between TBC in logarithm and permittivities at a single frequency, chemometrics was used to reduce noise, identify outliers, select effective variables, and divide sample sets. Several linear models, such as multiple linear regression, ridge regression, and least absolute shrinkage and selection operator, were established to determine TBC based on the effective spectra of $\varepsilon^{\prime}, \varepsilon^{\prime \prime}$, and their combination $\left(\varepsilon^{\prime}+\varepsilon^{\prime \prime}\right)$. The results indicated that the models built using the spectra of $\varepsilon^{\prime}+\varepsilon^{\prime \prime}$ and $\varepsilon^{\prime}$ had excellent TBC prediction performance. The best model was multiple linear regression based on $\varepsilon^{\prime}+\varepsilon^{\prime \prime}$ spectra with the residual predictive deviation of 3.26 . This study shows that the dielectric spectra had great potential to quantitatively and rapidly determine TBC of raw milk.

Key words: milk, total bacterial count, determination, dielectric spectroscopy
\end{abstract}

\section{INTRODUCTION}

Milk has various essential nutrients such as minerals, vitamins, and easily digestible proteins with balanced AA profiles that are significant in sustaining most body functions. Hygienic profile, characterized by contamination levels and distributions of microorganisms, is a critical parameter of raw milk quality (Zucali et al., 2011). Milk is almost sterile when secreted into the alveoli of the udder. After secretion, bacteria can enter milk from a variety of possible sources. High bacterial counts suggest the existence of microbial contamina-

Received March 21, 2019.

Accepted May 12, 2019.

*Corresponding author: guowenchuan69@126.com tion. The inside of the udder subsequent to synthesis, the outside of the udder, and the surface of milk processing and storage equipment are the sources of bacteria (Kelly et al., 2009). Total bacterial count (TBC) is the measure of the number of bacteria grown with the specified culture medium and temperature over a fixed period of time (Blowey and Edmondson, 2010). Standard plate count is the analytical reference method to determine the TBC. Although this method is relatively economical, sensitive, and used as a gold standard, the main defects of this method are labor intensiveness and long analysis time.

With the development of technology, various rapid detection methods have been applied in the microbial analysis. Molecular methods, which detect the genomic elements (DNA or RNA), such as direct epifluorescent filter technique (Hermida et al., 2000), flow cytometry (García Carrillo et al., 2018), and PCR (Lahtinen et al., 2006), could be viable alternatives to traditional methods. In addition, impedance measurement (Radhakrishnan et al., 2014), biosensors (Huang et al., 2016), and near-infrared spectroscopy (Numthuam et al., 2017) have been studied. Due to high specificity and sensitivity, biosensors could detect a broad spectrum of analytes in complex sample matrices and give real-time results without pre-enrichment. Several rapid enumeration methods are commercially available for the detection of microorganisms, such as fluorescent microscopy (Woźniak et al., 2018), quantitative PCR (Turgay et al., 2016), and flow cytometry (Raymond and Champagne, 2015). The results can be quickly and accurately obtained based on these techniques. However, due to the expensive instruments and the high costs of acquisition and operation of systems, these technologies are used only in large analytical laboratories and companies.

As a nondestructive technology, dielectric spectroscopy is an inexpensive method in composition analysis that supports in situ and on-line quality determination. Recently, several studies have investigated the feasibility of dielectric spectra in the determination of internal qualities such as fat (Zhu et al., 2018a) and protein (Zhu et al., 2016) of raw cow milk. Cow milk and goat 
milk are the 2 important types of milk in dairy production. Shaanxi Province, known as "the town of Chinese milk goat," is the largest milk goat production base in China. However, due to the small-scale farming of goats in many areas of Shaanxi, raw goat milk quality cannot be guaranteed. Therefore, developing a rapid method for detecting TBC of raw goat milk is very important for the local goat milk industry.

Dielectric properties, including dielectric constant $\left(\varepsilon^{\prime}\right)$ and dielectric loss factor $\left(\varepsilon^{\prime \prime}\right)$, are applied to explain the interactions of materials with electric fields. The former reflects the ability of a sample in storing electric energy, and the latter shows the ability of the material in dissipating electrical energy into heat (Skierucha et al., 2012). As microorganisms grow and nutrients metabolize, large molecules change to smaller molecules in a liquid system and cause changes in electrical conductivity and resistance in the liquid as well as at the interphase of electrodes (Fung, 1994). The changes can significantly affect the response of materials to electromagnetic fields. Our previous studies showed that dielectric spectra combined with chemometric methods have great potential in qualitatively predicting hygienedisqualified raw goat milk from qualified milk (Zhu et al., 2018b). Because the TBC had a wide change range, quantitatively determining TBC is very important for food quality control. To our knowledge, no work has been conducted on quantitative analysis of TBC using dielectric spectra. Therefore, we used raw goat milk to (1) investigate the relationship between TBC and dielectric properties, (2) extract effective variables from dielectric spectra, (3) establish different linear models for determining TBC, and (4) evaluate the potential of dielectric spectra as a means of quantitatively determining TBC of milk.

\section{MATERIALS AND METHODS}

\section{Sample Preparation}

Fresh raw milk samples were obtained manually from healthy Saanen goats at 5 local small-scale farms in Yangling, Shaanxi, China. Each milk sample was kept in a sterilized plastic bottle and delivered to the laboratory in a natural state within 10 min after milking. Upon arrival, each milk sample was stirred for about 1 min using an electric blender (OA2000, Shanghai Ouhor Equipment Co., Shanghai, China) to guarantee the uniform distribution of components. Then, it was divided into 3 groups to perform different tests, including chemical analyses, TBC determination, and dielectric spectra acquisition. These tests were conducted immediately and in parallel after the samples were divided. Each milk sample was used within $5 \mathrm{~h}$.
During the experiments, all milk samples were kept at room temperature $\left(24 \pm 1^{\circ} \mathrm{C}\right)$. A total of 154 samples were obtained from early lactation to the end of lactation of goats in 2016.

\section{Methods Used to Measure Milk Composition, TBC, and Dielectric Spectra}

Milk Composition. The composition of the obtained raw goat milk samples, including protein, fat, lactose, and SNF content, was measured using a Lactoscan milk analyzer (S60, Milkotronic Ltd., Nova Zagora, Bulgaria). The moisture content was determined by referring to Association of Official Analytical Chemists method 927.05 (AOAC International, 2000), and the $\mathrm{pH}$ value was obtained using a $\mathrm{pH}$ meter (PHSJ3F, Shanghai Precision Science Instrument Co. Ltd., Shanghai, China). All analyses were performed at room temperature $\left(24 \pm 1^{\circ} \mathrm{C}\right)$.

Determination of $T B C$. The plate count method was applied to measure TBC according to Chinese national standard GB4789.2-2010 (Ministry of Health of the People's Republic of China, 2010b). The processes are described in detail by Zhu et al. (2018b). The mean value of triplicate was regarded as the result of the TBC of each milk sample. To provide normal distribution and reduce skewness, the TBC was logarithmically transformed $\left(\log _{10}\right)$. Therefore, the unit of the TBC in this study was $\log _{10}$ colony-forming units per milliliter.

Dielectric Spectra Acquisition. The measurement system of dielectric spectra mainly consisted of an Agilent E5071C vector network analyzer (Agilent Technologies, Penang, Malaysia), an Agilent 85070E open-ended coaxial line probe, and other accessories. The frequency range of the network analyzer was set from 20 to 4,500 MHz, and the sampling points in the logarithmic scale were 201 . The permittivities, such as $\varepsilon^{\prime}$ and $\varepsilon^{\prime \prime}$, were calculated according to the reflection coefficient at the material-probe interface. The detailed information related to the setup and the calibration procedure of the dielectric spectra acquisition system could be found elsewhere (Zhu et al., 2016).

Before spectra acquisition, the system was warming up for at least $1 \mathrm{~h}$ to keep dielectric data steady, followed by calibrating the network analyzer and coaxial line probe. After each milk sample was stirred for $1 \mathrm{~min}$, the sample was moved into three $10-\mathrm{mL}$ beakers with an inner diameter of $22.5 \mathrm{~mm}$ and a depth of $25 \mathrm{~mm}$. The beakers were heated using a constant-temperature water bath. After the sample temperature increased to $25^{\circ} \mathrm{C}$ in no more than 2 min and remained stable, 2 original dielectric spectra (i.e., $\varepsilon^{\prime}$ and $\varepsilon^{\prime \prime}$ spectra) at 201 discrete frequencies were measured in about $30 \mathrm{~s}$. Three measurements were taken on the milk sample in 
each beaker, and 3 replications were conducted for each sample. Finally, the mean value of 9 measurements for each milk sample was calculated and used for subsequent analyses.

\section{Spectral Preprocessing and Analysis Methods}

Data Preprocessing. In addition to useful information, original spectra always contain noises. The spectral data should be preprocessed before further processing to get reliable, accurate, and stable results. Savitzky-Golay (S-G) smoothing is able to diminish high-frequency noises in raw spectra, and standard normal variate $(\mathbf{S N V})$ can separate physical variances from chemical ones in spectra (Afseth et al., 2006). Therefore, the original dielectric spectra were preprocessed by the combination of S-G smoothing and SNV in this study. Detailed descriptions on S-G smoothing and SNV can be found in other articles (Savitzky and Golay, 1964; Rinnan et al., 2009).

Outlier Detection. Because the presence of outliers in a sample set can significantly influence subsequent analysis results, outliers are usually eliminated from the sample set. Mahalanobis distance (MD) automatically finds the samples that are far from the center of the sample set (Mahalanobis, 1936). However, the high correlations between variables at different frequencies make the variance-covariance matrix used in MD too ill-conditioned for inversion (Thennadil et al., 2018). In this study, principal component (PC) analysis was used to obtain an orthogonal scores matrix of the spectral data for the calculation of MD. The number of PC was determined by the total contribution rate of the chosen PC. According to Pauta criterion ( $\mathrm{Li}$ et al., 2016), the sum of 3 times standard deviation and mean value of MD was used as the threshold of MD in this study.

Effective Variable Selection. Much redundant information contained in spectra usually causes negative influences on the accuracy and robustness of a calibration model. Variable importance in prediction (VIP) score, which is able to understand $\mathbf{X}$ space predictor variables that best explain $\mathbf{y}$ variance, was used as the effective variable selection method in this study. The average of the squared VIP scores equals 1 . Therefore, 1 is generally used as the threshold in effective variable selection.

Sample Division. To obtain a subset that can uniformly and sufficiently reflect the whole sample space, sample division methods are usually used to select the maximally diverse subset in a big sample set. The method used for sample division in this study was the joint $\mathrm{x}$-y distances (SPXY), which considers spectra and reference values together. Detailed information about SPXY can be found elsewhere (Galvão et al., 2005).

Latent Variable Extraction. Partial least squares (PLS) regression is a way to extract latent variables $(\mathbf{L V})$ as new eigenvectors of the spectra for the dimensionality reduction and data compression. The method is particularly effective when a lot of correlations or colinearities are presented in spectral data of biological material (Nicolaï et al., 2007). In this study, the extracted LV based on dielectric spectra were applied as the inputs of models to reduce the dimension of inputs and improve the speed and stability of established models.

\section{Modeling Methods and Model Assessment}

In this study, multiple linear regression (MLR), ridge regression (RR), and least absolute shrinkage and selection operator (LASSO) were used to establish TBC quantitative analysis models.

MLR Model. In the MLR model, the output variables $\mathbf{y}$ are explained by a linear combination of input variables $\mathbf{X}$ as follows:

$$
\mathbf{y}=\mathbf{X} \boldsymbol{\beta},
$$

where $\boldsymbol{\beta}$ is the regression coefficient matrix and is estimated by the ordinary least squares method, which finds the solution to minimize the residual sum of squares (RSS) as

$$
\mathrm{RSS}_{\mathrm{MLR}}=\|\hat{\mathbf{y}}-\mathbf{X} \boldsymbol{\beta}\|^{2}
$$

where $\hat{\mathbf{y}}$ is the predicted values vector of the output variables. The estimated regression coefficient matrix could be expressed as

$$
\hat{\boldsymbol{\beta}}_{\mathrm{MLR}}=\left(\mathbf{X}^{T} \mathbf{X}\right)^{-1} \mathbf{X}^{T} \mathbf{y}
$$

where $T$ is the symbol of transposition, Because the high colinearity presented in the spectral data readily leads to the overfitting and reduction of robustness, the MLR model may not perform well in many situations (Wang et al., 2015).

$R R$ Model. The RR model prevents overfitting and underfitting by introducing a penalizing term, $\alpha\|\beta\|_{2}^{2}$, in the RSS of the MLR model. The regression coefficient matrix is calculated as follows:

$$
\hat{\boldsymbol{\beta}}_{\mathrm{RR}}=\left(\mathbf{X}^{T} \mathbf{X}+\alpha \mathbf{I}\right)^{-1} \mathbf{X}^{T} \mathbf{y},
$$


where $\alpha$ is the ridge parameter and $\mathbf{I}$ is the identity matrix. Appropriate $\alpha$ can improve regression condition and reduce variance of estimates (Deng et al., 2018). The choice of $\alpha$ is often conducted using a $k$-fold cross-validation approach. Compared with MLR, RR frequently obtains a smaller mean squared error.

LASSO Model. The LASSO model is the least squares method with an $\mathrm{L}_{1}$ constraint on the regression parameters. The penalizing term, $\lambda\|\beta\|_{1}$, is introduced in the RSS of the MLR model. The regression coefficient of each input variable can be obtained by Equation 5:

$$
\hat{\beta}_{j}^{\mathrm{LASSO}}=\hat{\beta}_{j}^{\mathrm{MLR}} \max \left(0,1-\frac{n \lambda}{\left|\hat{\beta}_{j}^{\mathrm{MLR}}\right|}\right),
$$

where $j$ is the index of variables, $\lambda$ is a parameter of LASSO, and $n$ is the number of samples. The optimal value $\lambda$ is also chosen by using a $k$-fold cross-validation approach. In contrast with RR, LASSO shrinks some coefficients and sets others to 0 . Therefore, LASSO has the advantage in estimating parameters and selecting variables simultaneously (Hsu et al., 2008).

Model Performance Assessment. The correlation coefficient of calibration set $\left(\mathbf{r}_{\mathbf{c}}\right)$, correlation coefficient of prediction set $\left(\mathbf{r}_{\mathbf{p}}\right)$, root mean squared error of the calibration set (RMSEC), root mean squared error of the prediction set (RMSEP), and residual predictive deviation (RPD) were calculated for the evaluation of models. The RPD was defined as the ratio of the standard deviation of the $y$-variables to the RMSEP. An RPD value from 2.5 to 3.0 or greater than 3.0 indicates that the model has good or excellent prediction accuracy. An RPD value between 2.0 and 2.5 means that the model could be used to predict roughly (Nicolaï et al., 2007).

Software. Matlab version 7.1 (The Mathworks Inc., Natick, MA) was applied to preprocess the dielectric spectra, divide samples into calibration and prediction sets, and reduce spectral data. Spyder 3.2.6 (https:// www.spyder-ide.org) was applied to establish the MLR, RR, and LSAAO models.

\section{RESULTS AND DISCUSSION}

\section{Statistical Analyses of Milk Samples}

The statistical results of the milk composition, $\mathrm{pH}$, and TBC of 154 raw milk samples are shown in Table 1. The fat, protein, lactose, SNF, and water contents were $4.01 \pm 0.88,3.74 \pm 0.14,3.57 \pm 0.19,7.24 \pm 0.99$, and $88.71 \pm 1.55$, respectively. The $\mathrm{pH}$ values were 6.48 \pm 0.19 . Because the milk samples were collected from different local farms and from different periods of lactation, milk composition largely varied. The TBC of the milk samples ranged from 2.61 to $10.53 \log _{10} \mathrm{cfu} / \mathrm{mL}$, and the mean value and standard deviation were 6.47 and $1.93 \log _{10} \mathrm{cfu} / \mathrm{mL}$, respectively. If the TBC of the milk was greater than $6.30 \log _{10} \mathrm{cfu} / \mathrm{mL}\left(2 \times 10^{6} \mathrm{cfu} /\right.$ $\mathrm{mL}$ ), the milk was regarded as hygiene disqualified in accordance with Chinese national standard GB193012010 (Ministry of Health of the People's Republic of China, 2010a). In this study, 80 milk samples were hygiene qualified and 74 were hygiene disqualified. Table 1 shows the Pearson correlation coefficient and the $P$-value of each milk component and $\mathrm{pH}$ with TBC. Among the investigated compositions and $\mathrm{pH}$, only $\mathrm{pH}$ was highly correlated $(P<0.01)$ with TBC, but its Pearson correlation coefficient was only -0.503 . The poor correlation coefficients of the milk components and $\mathrm{pH}$ with $\mathrm{TBC}$ indicate that the milk composition and $\mathrm{pH}$ had difficulty predicting the TBC of milk.

\section{Dielectric Spectra of Milk Samples}

Figure 1 shows the original $\varepsilon^{\prime}$ spectra and $\varepsilon^{\prime \prime}$ spectra of 154 raw goat milk samples: $\varepsilon^{\prime}$ decreased with the increase of frequency over the whole frequency range and decreased rapidly below $40 \mathrm{MHz}$, and $\varepsilon^{\prime \prime}$ decreased with increasing frequency below about 2,000 $\mathrm{MHz}$ and

Table 1. Statistical results on composition, pH, and total bacterial count (TBC) of used raw goat milk samples and the correlation analysis of composition and $\mathrm{pH}$ with TBC

\begin{tabular}{|c|c|c|c|c|c|c|c|c|}
\hline Result & Parameter & $\begin{array}{c}\text { Fat, } \\
\%\end{array}$ & $\begin{array}{c}\text { Protein } \\
\%\end{array}$ & $\begin{array}{c}\text { Lactose } \\
\%\end{array}$ & $\begin{array}{c}\mathrm{SNF}, \\
\%\end{array}$ & $\begin{array}{c}\text { Moisture, } \\
\%\end{array}$ & $\mathrm{pH}$ & $\begin{array}{c}\text { TBC, } \\
\log _{10} \mathrm{cfu} / \mathrm{mL}\end{array}$ \\
\hline \multirow[t]{3}{*}{ Statistic } & Minimum & 1.90 & 3.45 & 3.29 & 2.57 & 77.01 & 5.73 & 2.61 \\
\hline & Maximum & 6.57 & 4.35 & 5.35 & 8.73 & 90.76 & 6.78 & 10.53 \\
\hline & SD & 0.88 & 0.14 & 0.19 & 0.99 & 1.55 & 0.19 & 1.93 \\
\hline \multirow[t]{2}{*}{ Correlation analysis } & Pearson's coefficient & +0.122 & -0.090 & -0.022 & +0.082 & +0.002 & -0.503 & $\mathrm{ND}^{1}$ \\
\hline & $P$-value & 0.133 & 0.269 & 0.790 & 0.310 & 0.981 & $<0.001$ & ND \\
\hline
\end{tabular}

${ }^{1}$ Analysis was not done. 
then increased with the increase of frequency above 2,000 MHz. Similar effects of frequency on $\varepsilon^{\prime}$ and $\varepsilon^{\prime \prime}$ of raw milk have been found in several studies (Zhu et al., 2016, 2018a).

At low frequency range, an electric dipole can freely follow the direction change of the electric field. This kind of polarization is able to store energy. When the frequency increases due to inertia, the dipole motion will not be able to keep up with the change of field. This causes a reduction in the energy storage of the electric field and an increase in energy losses due to the rotation (Skierucha et al., 2012). Therefore, $\varepsilon^{\prime}$ decreased with increasing frequency over the whole frequency range and $\varepsilon^{\prime \prime}$ slightly increased with increasing frequency after 2,000 MHz. Various charged ions are dissolved in milk. The electrical field makes dissolved ions move toward oppositely charged regions. This type of electric current is the reason for energy loss, and it typically predominates at the frequency below 1,000 MHz (Ryynänen, 1995). Some studies have also reported that the decrease of $\varepsilon^{\prime \prime}$ with the increase of frequency below a certain frequency is caused by ionic conduction (Nelson, 2005; Zhu and Guo, 2017).

\section{Correlation Between Dielectric Properties and TBC}

After the original dielectric spectra were preprocessed by SNV after S-G smoothing, the scatter plots of dielectric properties $\left(\varepsilon^{\prime}\right.$ and $\left.\varepsilon^{\prime \prime}\right)$ against TBC were drawn to find their relationship at each frequency over the investigated frequency range. Figure 2 shows the obtained $\varepsilon^{\prime}$ and $\varepsilon^{\prime \prime}$ against TBC at $50 \mathrm{MHz}$. We tried to regress their relationships using linear or nonlinear

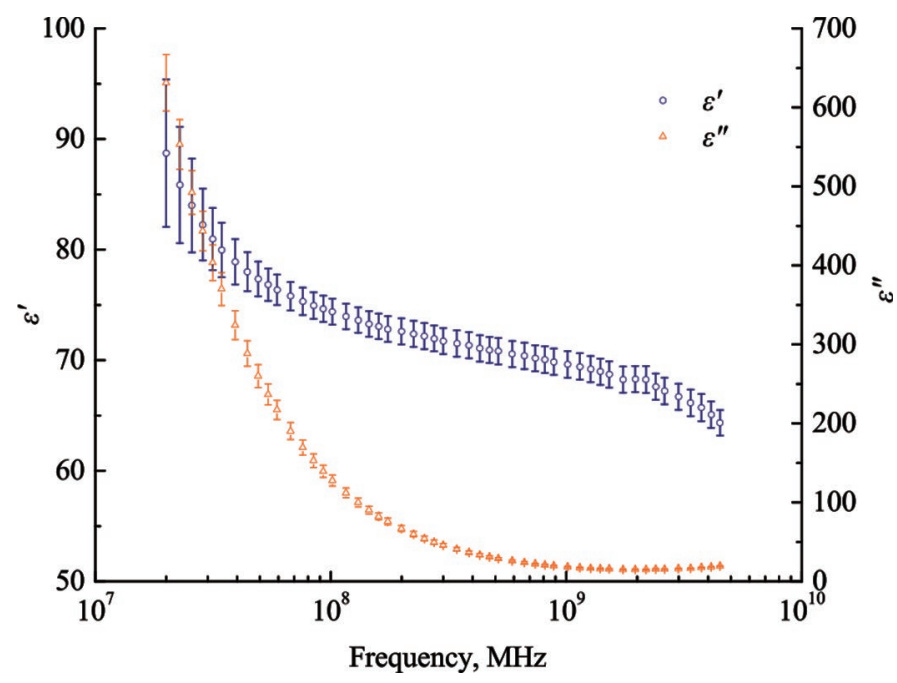

Figure 1. Original dielectric constant $\left(\varepsilon^{\prime}\right)$ spectra and dielectric loss factor $\left(\varepsilon^{\prime \prime}\right)$ spectra of 154 raw goat milk samples.

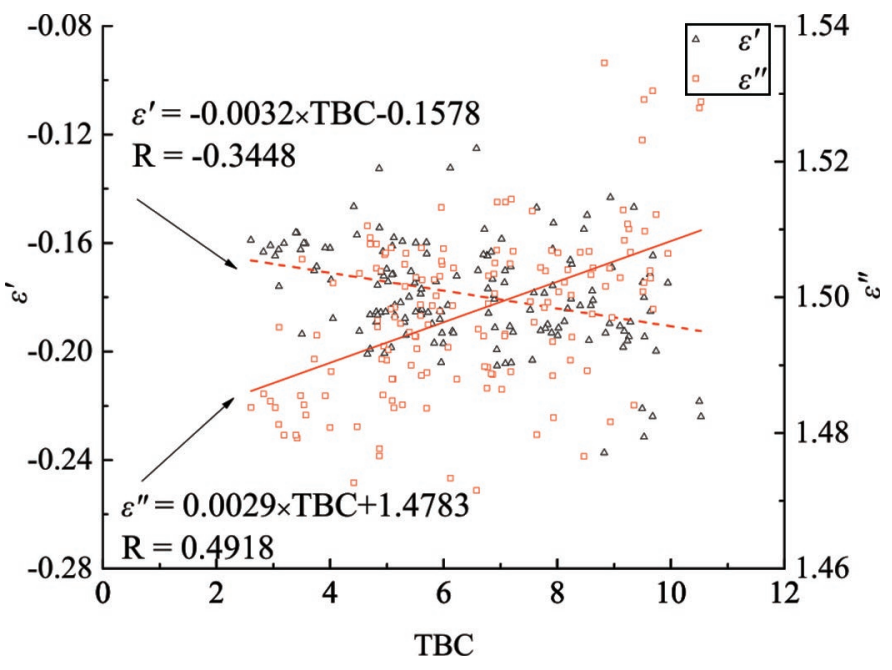

Figure 2. The dielectric constant $\left(\varepsilon^{\prime}\right)$ and dielectric loss factor $\left(\varepsilon^{\prime \prime}\right)$ against total bacterial count (TBC) at $50 \mathrm{MHz}$.

models. Figure 3 shows the linear correlation coefficient (r) between $\varepsilon^{\prime}$ and $\varepsilon^{\prime \prime}$ with TBC at 201 frequencies from 20 to $4,500 \mathrm{MHz}$. It was found that the $\mathrm{r}$ for $\varepsilon^{\prime}$ almost remained at -0.50 when the frequency was above about $100 \mathrm{MHz}$. The $\mathrm{r}$ for $\varepsilon^{\prime \prime}$ changed between 0.41 and 0.50 above about $50 \mathrm{MHz}$. Therefore, the linear relationship was not good enough to allow accurate calculation of TBC based on the $\varepsilon^{\prime}$ and $\varepsilon^{\prime \prime}$ at a single frequency. Moreover, the nonlinear models that could describe the relationship between permittivities and TBC at a single frequency well had not been found.

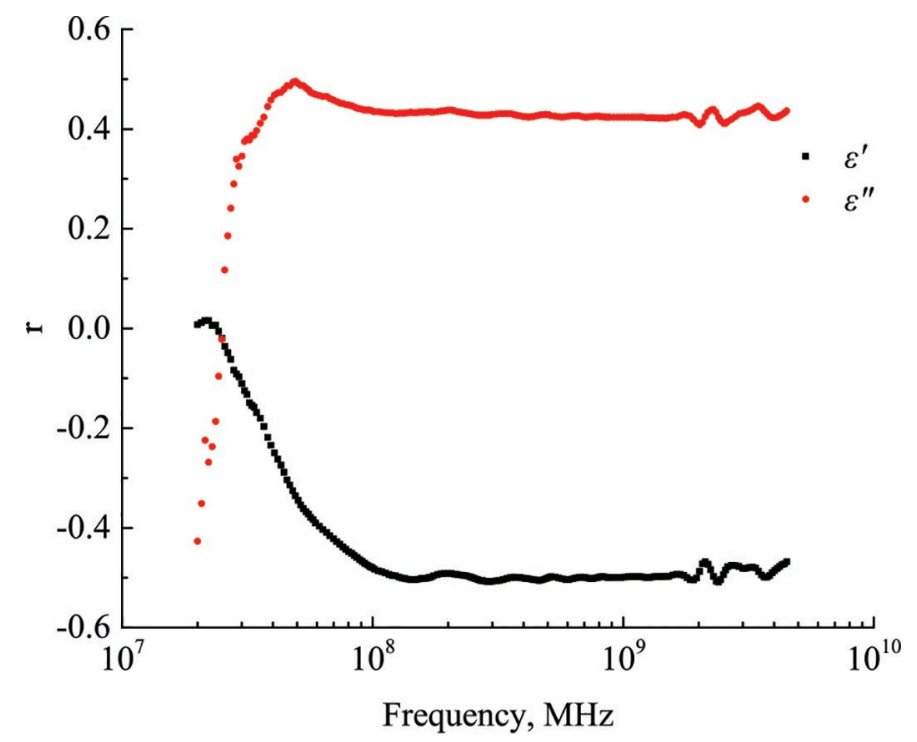

Figure 3. The correlation coefficients $(\mathrm{r})$ of linear relationships between dielectric constant $\left(\varepsilon^{\prime}\right)$ and dielectric loss factor $\left(\varepsilon^{\prime \prime}\right)$ and total bacterial count at 201 frequencies from 20 to $4,500 \mathrm{MHz}$. 


\section{Outlier Detection}

The dielectric spectra after preprocessing were turned into an orthogonal scores matrix by $\mathrm{PC}$ analysis. The first $3 \mathrm{PC}$, whose total contribution rate was greater than $99 \%$, were used as the substitute of the dielectric spectra to calculate the MD. The obtained MD of all samples were between 0.29 and 4.33 , and the mean value and standard deviation of MD were 1.52 and 0.82 , respectively. The determined threshold was 4.00 according to Pauta criterion. Finally, 3 samples were identified as outliers, and 151 samples were used for later analysis.

\section{Effective Variable Selection}

The PLS models at different numbers of LV from 1 to 20 with an interval of 1 were established based on preprocessed dielectric spectra of 151 milk samples by applying the leave-one-out cross-validation method. The PLS model with $16 \mathrm{LV}$, which made the root mean squared error of cross-validation (RMSECV) the lowest, was chosen to calculate the VIP scores. The VIP scores of 402 dielectric variables were between 0.55 and 3.90. An appropriate threshold of VIP score is crucial for selecting effective variables that contribute most to the underlying variation in the variables. Usually, the greater-than-one rule is used as a criterion for variable selection, but some studies reported that useful information was eliminated or irrelevant information



Figure 4. Total bacterial count (TBC) distributions of milk samples in the calibration set and prediction set divided by joint $\mathrm{x}-\mathrm{y}$ distances. was included with this criterion (Tran et al., 2014). Therefore, in this study, the thresholds were set as 0.4 , $0.6,0.8,1.0,1.2$, and 1.4 , and the determined numbers of effective variables were 402 (all variables), 351, 208, 122,58 , and 31 . Based on the selected effective variables, the leave-one-out cross-validation method was used to calculate the RMSECV, and the results were $1.08,1.08,1.10,1.20,1.13$, and 1.14 , respectively. No big difference was found on the values of RMSECV when the effective variables determined by VIP of 0.4 , 0.6 , and 0.8 were used as inputs. Because the number of effective variables of 351 , determined by VIP of 0.6 , was much larger than the number of 208 determined by the VIP of $0.8,0.8$ was applied as the threshold of VIP. Finally 208 variables, including 101 variables of $\varepsilon^{\prime}$ and 107 variables of $\varepsilon^{\prime \prime}$, were determined as effective dielectric variables. The $\varepsilon^{\prime}$ spectra (101 variables), $\varepsilon^{\prime \prime}$ spectra (107 variables), and their combination $\left(\varepsilon^{\prime}+\varepsilon^{\prime \prime}\right.$ spectra; 208 variables) were applied in subsequent sample division and modeling.

\section{Sample Division}

In this study, 151 normal samples were divided into calibration and prediction sets using SPXY algorithm according to the ratio of $4: 1$. Therefore, 121 samples were separated into the calibration set and the remaining 30 samples were divided into the prediction set. Figure 4 indicates the TBC distributions of milk samples in the calibration and prediction sets. The 2 distributions were very similar, indicating that the sample division in this study was reasonable.

\section{Modeling Results}

Determination of $L V$. The number of $L V$ is an important parameter in establishing the linear models of MLR, RR, and LASSO models. To determine the optimal number of LV, the RMSECV of PLS models were calculated when the number of LV changed from 1 to 20 with an increment of 1 and when the $\varepsilon^{\prime}$ spectra, $\varepsilon^{\prime \prime}$ spectra, and $\varepsilon^{\prime}+\varepsilon^{\prime \prime}$ spectra were used as inputs of PLS, respectively. The results are shown in Figure 5, which shows that the RMSECV decreased quickly with increased LV number when the number of LV was small. With the continuous increase of LV number, the RMSECV changed differently. The lowest RMSECV were found at the LV numbers of 20,15 , and 14 when the $\varepsilon^{\prime}$, $\varepsilon^{\prime \prime}$, and $\varepsilon^{\prime}+\varepsilon^{\prime \prime}$ spectra were used as inputs, respectively. Therefore, 20, 15, and 14 were the optimal numbers of LV for $\varepsilon^{\prime}, \varepsilon^{\prime \prime}$, and $\varepsilon^{\prime}+\varepsilon^{\prime \prime}$ spectra and were used to build MLR, RR, and LASSO models. The only output of each model was the TBC of milk. 


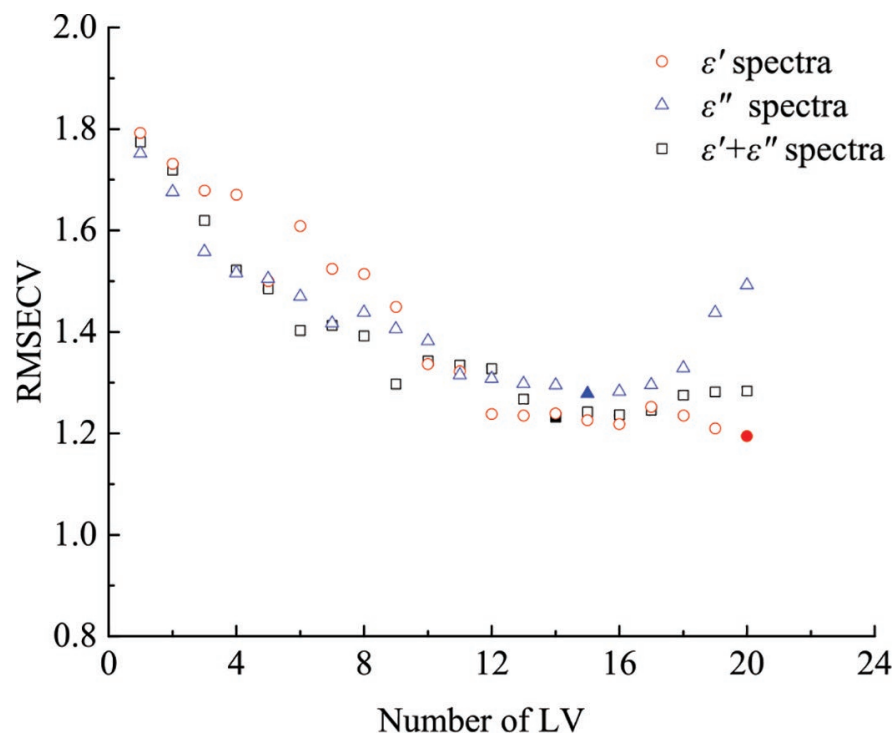

Figure 5. Calculated root mean squared error of cross-validation (RMSECV) of the partial least squares model at different numbers of latent variable (LV) from 1 to 20 based on the spectra of dielectric constant $\left(\varepsilon^{\prime}\right)$, dielectric loss factor $\left(\varepsilon^{\prime \prime}\right)$, and $\varepsilon^{\prime}+\varepsilon^{\prime \prime}$.

MLR Modeling Results. Table 2 lists the TBC determination results for 151 milk samples in the calibration and prediction sets using MLR models. For the calibration set, the MLR model established using $\varepsilon^{\prime}$ spectra had the highest $\mathrm{r}_{\mathrm{c}}(0.91)$ and the lowest RMSEC $\left(0.79 \log _{10} \mathrm{cfu} / \mathrm{mL}\right)$ compared with that built using $\varepsilon^{\prime \prime}$ spectra $\left(\mathrm{r}_{\mathrm{c}}=0.85 ; \mathrm{RMSEC}=0.98 \log _{10} \mathrm{cfu} / \mathrm{mL}\right)$ and $\varepsilon^{\prime}+\varepsilon^{\prime \prime}$ spectra $\left(\mathrm{r}_{\mathrm{c}}=0.88 ; \mathrm{RMSEC}=0.89 \log _{10} \mathrm{cfu} /\right.$ $\mathrm{mL})$. For the prediction set, the MLR model built with $\varepsilon^{\prime}+\varepsilon^{\prime \prime}$ spectra had the lowest RMSEP (0.58 $\log _{10} \mathrm{cfu} /$ $\mathrm{mL}$ ) compared with that established using $\varepsilon^{\prime}$ spectra $\left(\mathrm{RMSEP}=0.61 \log _{10} \mathrm{cfu} / \mathrm{mL}\right)$ and $\varepsilon^{\prime \prime}$ spectra (RMSEP $\left.=0.84 \log _{10} \mathrm{cfu} / \mathrm{mL}\right)$. That is, the MLR model had the best calibration performance when it was built with $\varepsilon^{\prime}$ spectra, whereas it had the best prediction performance when $\varepsilon^{\prime}+\varepsilon^{\prime \prime}$ spectra were used in modeling.

$R R$ Modeling Results. Determination of the optimal value of $\alpha$ is significant to get efficient and reliable RR models. In this study, $\alpha$ was set from 0.01 to 10 and increased by 0.01 from 0.01 . The optimal values of $\alpha$, determined by the lowest RMSECV using the 3 -fold cross-validation method, were $0.01,0.49$, and 0.61 when the RR models were built with $\varepsilon^{\prime}, \varepsilon^{\prime \prime}$, and $\varepsilon^{\prime}+\varepsilon^{\prime \prime}$ spectra, respectively. Table 2 shows the TBC determination performance of $\mathrm{RR}$ models based on the optimal $\alpha$ values and built using different dielectric spectra. The $\mathrm{RR}$ model built with $\varepsilon^{\prime}$ spectra had the highest $\mathrm{r}_{\mathrm{c}}(0.90)$ and $r_{p}(0.95)$ and the lowest RMSEC (0.81 $\log _{10} \mathrm{cfu} /$ $\mathrm{mL})$ and RMSEP $\left(0.61 \log _{10} \mathrm{cfu} / \mathrm{mL}\right)$.

LASSO Modeling Results. The optimal value of $\lambda$ in the LASSO model was decided by the lowest RMSECV using a 3-fold cross-validation approach when $\lambda$ was set from 0.01 to 10 with an interval of 0.01 . No matter which kind of spectra were used to build the LASSO model, the optimal value $\lambda$ was 0.01 . Table 2 also shows the TBC determination performance of LASSO models when $\lambda$ was 0.01 with different dielectric spectra as inputs. Clearly, the LASSO model built with $\varepsilon^{\prime}+\varepsilon^{\prime \prime}$ spectra had the highest $\mathrm{r}_{\mathrm{c}}(0.88)$ and the lowest RMSEC $\left(0.88 \log _{10} \mathrm{cfu} / \mathrm{mL}\right)$. Moreover, its performance in the prediction set $\left(\mathrm{r}_{\mathrm{p}}=0.94\right.$; RMSEP $=$ $0.66 \log _{10} \mathrm{cfu} / \mathrm{mL}$ ) was better than the LASSO model established with $\varepsilon^{\prime}$ spectra $\left(\mathrm{r}_{\mathrm{p}}=0.92 ; \mathrm{RMSEP}=0.76\right.$ $\left.\log _{10} \mathrm{cfu} / \mathrm{mL}\right)$ and $\varepsilon^{\prime \prime}$ spectra $\left(\mathrm{r}_{\mathrm{p}}=0.90 ; \mathrm{RMSEP}=0.83\right.$ $\left.\log _{10} \mathrm{cfu} / \mathrm{mL}\right)$. The results indicated that the LASSO model built with $\varepsilon^{\prime}+\varepsilon^{\prime \prime}$ spectra had the best calibration and prediction performances.

Table 2. Total bacterial count determination results of multiple linear regression (MLR), ridge regression (RR), and least absolute shrinkage and selection operator (LASSO) models based on different effective dielectric spectra ${ }^{1}$

\begin{tabular}{llccccc}
\hline $\begin{array}{l}\text { Modeling } \\
\text { method }\end{array}$ & $\begin{array}{l}\text { Dielectric } \\
\text { spectra }^{2}\end{array}$ & $\mathrm{r}_{\mathrm{c}}$ & $\begin{array}{c}\text { RMSEC, } \\
\log _{10} \mathrm{cfu} / \mathrm{mL}\end{array}$ & $\mathrm{r}_{\mathrm{p}}$ & $\begin{array}{c}\text { RMSEP, } \\
\log _{10} \mathrm{cfu} / \mathrm{mL}\end{array}$ & $\mathrm{RPD}$ \\
\hline MLR & $\varepsilon^{\prime}$ & 0.91 & 0.79 & 0.95 & 0.61 & 3.11 \\
& $\varepsilon^{\prime \prime}$ & 0.85 & 0.98 & 0.90 & 0.84 & 2.27 \\
$\mathrm{RR}$ & $\varepsilon^{\prime}+\varepsilon^{\prime \prime}$ & 0.88 & 0.89 & 0.95 & 0.58 & 3.26 \\
& $\varepsilon^{\prime}$ & 0.90 & 0.81 & 0.95 & 0.61 & 3.11 \\
LASSO & $\varepsilon^{\prime \prime}$ & 0.85 & 1.00 & 0.91 & 0.79 & 2.40 \\
& $\varepsilon^{\prime}+\varepsilon^{\prime \prime}$ & 0.88 & 0.88 & 0.94 & 0.65 & 2.95 \\
& $\varepsilon^{\prime}$ & 0.86 & 0.97 & 0.92 & 0.76 & 2.51 \\
& $\varepsilon^{\prime \prime}$ & 0.84 & 1.02 & 0.90 & 0.83 & 2.30 \\
\hline
\end{tabular}

${ }^{1} \mathrm{r}_{\mathrm{c}}=$ correlation coefficient of the calibration set; RMSEC = root mean squared error of the calibration set; $\mathrm{r}_{\mathrm{p}}$ $=$ correlation coefficient of the prediction set; RMSEP $=$ root mean squared error of the prediction set; RPD $=$ residual predictive deviation

${ }^{2} \varepsilon^{\prime}=$ dielectric constant; $\varepsilon^{\prime \prime}=$ dielectric loss factor. 


\section{DISCUSSION}

When the different spectra were used to build TBC determination models, $\varepsilon^{\prime}+\varepsilon^{\prime \prime}$ and $\varepsilon^{\prime}$ spectra made the models have excellent prediction performance (RPD > $2.5)$. However, the $\varepsilon^{\prime \prime}$ spectra only made the models have rough quantitative determination ability $(2.5>$ RPD > 2.0). Among the 9 built models, the MLR model based on $\varepsilon^{\prime}+\varepsilon^{\prime \prime}$ spectra had the best prediction ability, with an RPD of 3.26, followed by MLR (RPD = $3.11)$ and $\mathrm{RR}(\mathrm{RPD}=3.11)$ when $\varepsilon^{\prime}$ spectra were used to build TBC prediction models. Therefore, dielectric spectra have great potential in quantitatively determining TBC of raw goat milk.

Up until now, few studies have reported the TBC prediction by using quick spectra technologies for milk (Saranwong and Kawano, 2008; Numthuam et al., 2017). For example, Numthuam et al. (2017) applied near-infrared spectroscopy combined with bacterial cultivation (about $90 \mathrm{~min}$ ) to detect the TBC of raw milk, and they reported that the RMSEP of the PLS model was $0.32 \log _{10} \mathrm{cfu} / \mathrm{mL}$, which was superior to the best prediction performance obtained here $(\mathrm{RMSEP}=0.58$ $\log _{10} \mathrm{cfu} / \mathrm{mL}$ ). However, 90-min bacterial cultivation was needed when the TBC was predicted by Numthuam et al. (2017). Given the long time and complicated pretreatment necessary, the near-infrared spectroscopy method could not be used in the quick determination of TBC in milk. However, no bacterial cultivation and the direct dielectric properties measurement in milk indicate that the dielectric spectra technology can be applied in rapid detection of TBC in raw milk.

\section{CONCLUSIONS}

To evaluate the feasibility of dielectric spectra in the determination of TBC in raw milk, the dielectric spectra and TBC of 154 raw goat milk samples were measured using coaxial line probe technology and plate count technique, respectively. The SNV after S-G smoothing was used to reduce the noise of the original spectra. The MD was used to detect outliers, and 151 samples were selected as valid samples. A total of 208 variables, including $101 \varepsilon^{\prime}$ variables and $107 \varepsilon^{\prime \prime}$ variables, were selected as effective dielectric variables based on VIP scores. The selected effective spectra of $\varepsilon^{\prime}, \varepsilon^{\prime \prime}$, and $\varepsilon^{\prime}+\varepsilon^{\prime \prime}$ were used to establish MLR, RR, and LASSO models to quantitatively determine the TBC of milk. The spectra of $\varepsilon^{\prime}+\varepsilon^{\prime \prime}$ and $\varepsilon^{\prime}$ made the established models have excellent TBC determination, whereas the spectra of $\varepsilon^{\prime \prime}$ gave rough estimation for TBC. The best model was MLR $(\mathrm{RPD}=3.26)$ when $\varepsilon^{\prime}+\varepsilon^{\prime \prime}$ spectra were applied to build TBC prediction models, followed by MLR $(\mathrm{RPD}=3.11)$ and $\mathrm{RR}(\mathrm{RPD}=3.11)$ when $\varepsilon^{\prime}$ spectra were used to build TBC prediction models. The study shows that the dielectric spectra have great potential in quantitatively determining the TBC of raw milk in situ or in in-line detection.

\section{ACKNOWLEDGMENTS}

The authors gratefully acknowledge the financial support provided by the National Natural Science Foundation of China (project no. 31671935, Beijing).

\section{REFERENCES}

Afseth, N. K., V. H. Segtnan, and J. P. Wold. 2006. Raman spectra of biological samples: A study of preprocessing methods. Appl. Spectrosc. 60:1358-1367.

AOAC International. 2000. Official Methods of Analysis. 17th ed. Method: 927.05. AOAC International, Rockville, MD.

Blowey, R., and P. Edmondson. 2010. Bactoscan and total bacterial count (TBC). Pages 171-183 in Mastitis Control in Dairy Herds. R. Blowey and P. Edmondson, ed. CAB International, Wallingford, UK.

Deng, Y., H. Zeng, Y. Jiang, G. Chen, J. Chen, and L. Sun. 2018. Ridge regression for predicting elastic moduli and hardness of calcium aluminosilicate glasses. Mater. Res. Express 5:035205.

Fung, D. Y. C. 1994. Rapid methods and automation in food microbiology: A review. Food Rev. Int. 10:357-375.

Galvão, R. K. H., M. C. U. Araujo, G. E. José, M. J. C. Pontes, E. C. Silva, and T. C. B. Saldanha. 2005. A method for calibration and validation subset partitioning. Talanta 67:736-740.

García Carrillo, M., M. Ferrario, and S. Guerrero. 2018. Effectiveness of UV-C light assisted by mild heat on Saccharomyces cerevisiae $\mathrm{KE} 162$ inactivation in carrot-orange juice blend studied by flow cytometry and transmission electron microscopy. Food Microbiol. $73: 1-10$

Hermida, M., M. Taboada, S. Menéndez, and J. L. Rodríguezotero. 2000. Semi-automated direct epifluorescent filter technique for total bacterial count in raw milk. J. AOAC Int. 83:1345-1348.

Hsu, N.-J., H.-L. Hung, and Y.-M. Chang. 2008. Subset selection for vector autoregressive processes using Lasso. Comput. Stat. Data Anal. 52:3645-3657.

Huang, Y. M., H. Y. Hsu, and C. L. Hsu. 2016. Development of electrochemical method to detect bacterial count, Listeria monocytogenes, and somatic cell count in raw milk. J. Taiwan Inst. Chem. Eng. 62:39-44.

Kelly, P., K. O’Sullivan, D. P. Berry, S. J. More, W. J. Meaney, E. J. O'Callaghan, and B. O'Brien. 2009. Farm management factors associated with bulk tank total bacterial count in Irish dairy herds during 2006/07. Ir. Vet. J. 62:36-42.

Lahtinen, S. J., M. Gueimonde, A. C. Ouwehand, J. P. Reinikainen, and S. J. Salminen. 2006. Comparison of four methods to enumerate probiotic bifidobacteria in a fermented food product. Food Microbiol. 23:571-577.

Li, L., Z. Wen, and Z. Wang. 2016. Outlier detection and correction during the process of groundwater lever monitoring base on pauta criterion with self-learning and smooth processing. Pages 497-503 in Theory, Methodology, Tools and Applications for Modeling and Simulation of Complex Systems. L. Zhang, X. Song, and Y. Wu, ed. Springer Singapore, Singapore.

Mahalanobis, P. C. 1936. On the generalized distance in statistics. Proc. Nat. Inst. Sci. 2:49-55.

Ministry of Health of the People's Republic of China. 2010a. National food safety standard GB19301-2010. Raw milk. Ministry of Health of the People's Republic of China, Beijing, China.

Ministry of Health of the People's Republic of China. 2010b. National food safety standard GB4789.2-2010. Food microbiological exami- 
nation: Aerobic plate count. Ministry of Health of the People's Republic of China, Beijing, China.

Nelson, S. O. 2005. Dielectric spectroscopy of fresh fruit and vegetable tissues from 10 to $1800 \mathrm{MHz}$. J. Microw. Power Electromagn. Energy 40:31-47.

Nicolaï, B. M., K. Beullens, E. Bobelyn, A. Peirs, W. Saeys, K. I. Theron, and J. Lammertyn. 2007. Nondestructive measurement of fruit and vegetable quality by means of NIR spectroscopy: A review. Postharvest Biol. Technol. 46:99-118.

Numthuam, S., J. Hongpathong, R. Charoensook, and S. Rungchang. 2017. Method development for the analysis of total bacterial count in raw milk using near-infrared spectroscopy. J. Food Saf. 37:1-7.

Radhakrishnan, R., M. Jahne, S. Rogers, and I. I. Suni. 2014. Detection of Listeria monocytogenes by electrochemical impedance spectroscopy. Electroanalysis 25:2231-2237.

Raymond, Y., and C. P. Champagne. 2015. The use of flow cytometry to accurately ascertain total and viable counts of Lactobacillus rhamnosus in chocolate. Food Microbiol. 46:176-183.

Rinnan, Å., F. van den Berg, and S. B. Engelsen. 2009. Review of the most common pre-processing techniques for near-infrared spectra. Trends Analyt. Chem. 28:1201-1222.

Ryynänen, S. 1995. The electromagnetic properties of food materials: A review of the basic principles. J. Food Eng. 26:409-429.

Saranwong, S., and S. Kawano. 2008. System design for non-destructive near infrared analyses of chemical components and total aerobic bacteria count of raw milk. J. Near Infrared Spectrosc. 16:389-398.

Savitzky, A., and M. J. E. Golay. 1964. Smoothing and differentiation of data by simplified least squares procedures. Anal. Chem. $36: 1627-1639$.

Skierucha, W., A. Wilczek, and A. Szypłowska. 2012. Dielectric spectroscopy in agrophysics. Int. Agrophys. 26:187-197.

Thennadil, S. N., M. Dewar, C. Herdsman, A. Nordon, and E. Becker. 2018. Automated weighted outlier detection technique for multivariate data. Control Eng. Pract. 70:40-49.
Tran, T. N., N. L. Afanador, L. M. C. Buydens, and L. Blanchet. 2014. Interpretation of variable importance in partial least squares with significance multivariate correlation (smc). Chemom. Intell. Lab. Syst. 138:153-160.

Turgay, M., W. Schaeren, D. Wechsler, U. Bütikofer, and H. U. Graber. 2016. Fast detection and quantification of four dairy propionic acid bacteria in milk samples using real-time quantitative polymerase chain reaction. Int. Dairy J. 61:37-43.

Wang, H., J. Peng, C. Xie, Y. Bao, and Y. He. 2015. Fruit quality evaluation using spectroscopy technology: A review. Sensors (Basel) 15:11889-11927.

Woźniak, M., D. Połap, L. Kośmider, and T. Cłapa. 2018. Automated fluorescence microscopy image analysis of Pseudomonas aeruginosa bacteria in alive and dead stadium. Eng. Appl. Artif. Intell. 67:100-110.

Zhu, X., W. Guo, F. Kang, F. Kong, and Q. Zhu. 2016. Determination of protein content of raw fresh cow's milk using dielectric spectroscopy combined with chemometric methods. Food Bioprocess Technol. 9:2092-2102.

Zhu, X. H., W. C. Guo, D. Y. Liu, and F. Kang. 2018a. Determining the fat concentration of fresh raw cow milk using dielectric spectroscopy combined with chemometrics. Food Anal. Methods $11: 1528-1537$

Zhu, Z., and W. Guo. 2017. Frequency, moisture content, and temperature dependent dielectric properties of potato starch related to drying with radio-frequency/microwave energy. Sci. Rep. 7:9311.

Zhu, Z., X. Zhu, F. Kong, and W. Guo. 2018b. A rapid method on identifying disqualified raw goat's milk based on total bacterial count by using dielectric spectra. J. Food Eng. 239:40-51.

Zucali, M., L. Bava, A. Tamburini, M. Brasca, L. Vanoni, and A. Sandrucci. 2011. Effects of season, milking routine and cow cleanliness on bacterial and somatic cell counts of bulk tank milk. J. Dairy Res. 78:436-441. 